\title{
Analytical Evaluation of Nonlinear Effects on OFDMA Signals
}

\author{
Teresa Araújo and Rui Dinis, Member, IEEE
}

\begin{abstract}
As with other multicarrier modulations, OFDMA signals (Orthogonal Frequency Division Multiple Access) have large envelope fluctuations making them very prone to nonlinear distortion effects.

In this paper we study the impact of nonlinear devices on OFDMA signals. We present an analytical characterization of the signals at the output of a nonlinear device that can be used for obtaining the spectral characterization of the transmitted signals, as well as the computation of the nonlinear interference levels on the received signals.

It is shown that the power allocated to each user has a key impact on the nonlinear distortion effects. For this reason, we should avoid having low-power and high-power users in adjacent subcarriers. It is also shown that nonlinear distortion levels are lower when just a small fraction of the subcarriers is used (i.e., when the system load is small). ${ }^{1}$
\end{abstract}

Index Terms-OFDM signals, nonlinear effects, intermodulation analysis, wireless access, OFDMA.

\section{INTRODUCTION}

$\mathbf{O}$ FDMA (Orthogonal Frequency Division Multiple Access) schemes [1], [2] are OFDM (Orthogonal Frequency Division Multiplexing) modulations [3], [4] where a different set of subcarriers is assigned to each user. Therefore, they combine an OFDM modulation with an FDMA (Frequency Division Multiple Access) scheme. Moreover, they are suitable for severe frequency-selective channels (also denoted timedispersive) and allow a flexible and efficient management of the spectrum. For these reasons, they were selected for future broadband wireless systems [5]-[7]. OFDMA is used in wireless broadband access technologies IEEE 802.16a/d/e, commonly referred to as WiMAX [5], [8], and 3rd Generation Partnership Project (3GPP) Long Term Evolution (LTE), named High Speed OFDM Packet Access (HSOPA) [6]. It is also the access method candidate for Wireless Regional Area Networks (WRAN) [7].

As with OFDM and other multicarrier modulations, the transmitted signals have large envelope fluctuations and high PMEPR (Peak-to-Mean Envelope Power Ratio), leading to amplification difficulties. For this reason, several techniques

Manuscript received November 19, 2009; revised April 19, 2010; accepted June 17, 2010. The associate editor coordinating the review of this paper and approving it for publication was J. Olivier.

T. Araújo is with the Instituto de Telecomunicações, Lisboa, Portugal, and the Departamento de Matemática, Instituto Superior de Engenharia do Porto, Porto, Portugal (e-mail: tpa@isep.ipp.pt).

R. Dinis is with the Instituto de Telecomunicações, Lisboa, Portugal, and the Departamento de Engenharia Electrotécnica, Faculdade de Ciências e Tecnologia, FCT, Universidade Nova de Lisboa, Caparica, Portugal (e-mail: rdinis@fct.unl.pt).

Digital Object Identifier 10.1109/TWC.2010.081810.091662

${ }^{1}$ This work was partially published in IEEE $5^{\text {th }}$ International Symposium on Wireless Communication Systems 2008 (ISWCS'08) and IEEE $67^{\text {th }}$ Vehicular Technology Conference 2008 (VTC'08 Spring). have been proposed to reduce the envelope fluctuations of OFDMA signals namely through suitable pre-processing schemes [9]-[11]. As an alternative, we can employ clipping and filtering techniques, already shown to be effective for conventional OFDM signals [12]-[14], as well as MC-CDMA (Multi-Carrier Code Division Multiple Access) schemes [15].

The performance evaluation of multicarrier schemes with nonlinear transmission (either due to an imperfectly linear amplification or due to suitable signal processing schemes to reduce the envelope fluctuations of the transmitted signals), usually resorts to Monte-Carlo simulations that require a long computation time; heuristic, "semi-analytical", approaches have also been proposed so as to evaluate nonlinear distortion effects (see, e.g., [16]). When the number of subcarriers is high we can take advantage of the Gaussian nature of the transmitted signals to characterize statistically the transmitted signals [12], [15], [17]-[20]. The nonlinear devices considered in [12], [15], [17], [18] can be regarded as bandpass memoryless nonlinearities [21] ([15] considers MC-CDMA signals and [12], [17], [18] consider OFDM signals). [19], [20] consider quantization effects on OFDM signals, i.e., the nonlinear devices can be regarded as I-Q memoryless nonlinearities.

This paper deals with the analytical evaluation of nonlinear distortion effects on OFDMA signals, which, as far as we know, was not addressed in detail in the literature. We consider nonlinear distortion effects that are inherent to nonlinear signal processing techniques for reducing the PMEPR of the transmitted signals (as the ones proposed in [12] for conventional OFDM schemes). For this purpose, we take advantage of the Gaussian-like nature of OFDMA signals with a large number of subcarriers to extend the results of [12], [17], [22] to OFDMA schemes. Our results allow an analytical spectral characterization of the transmitted signals, as well as the computation of the nonlinear interference levels on the received signals. They can also be used to compute the corresponding BER (Bit Error Rate). This allows an efficient approach for studying aspects such as the type of nonlinear device, the impact of the system load (fraction of subcarriers used), the set of subcarriers assigned to each user (continuous, randomly spaced or regularly spaced subcarriers), etc.

This paper is organized as follows: Sec. II describes the OFDMA schemes considered in this paper. The analytical evaluation of nonlinear distortion effects on the transmission of OFDMA signals is made in Sec. III and a set of performance results is presented in Sec. IV. Finally, Sec. V is concerned with the conclusions of this paper.

\section{OFDMA SYSTEM}

In this paper we consider an OFDMA system with $P$ users and $N_{p}$ subcarriers assigned to the $p$ th user. The total number 

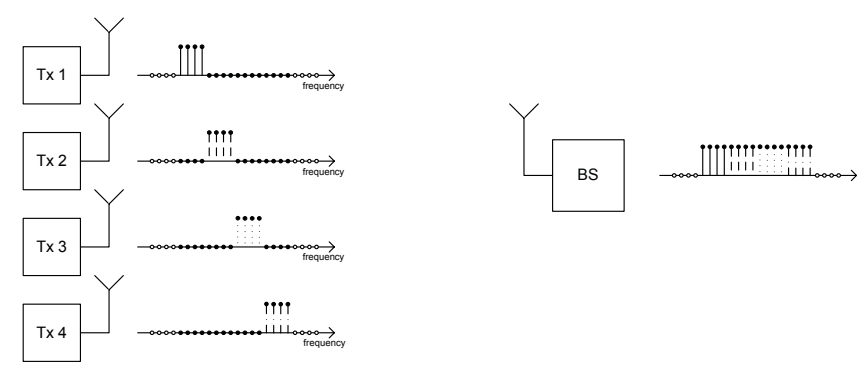

Fig. 1. OFDMA uplink system with $p$ users.

of subcarriers is $N^{\prime}$, the number of in-band subcarriers (i.e., the number of subcarriers that can be assigned to users) is $N$ (it is assumed that $N \geq \sum_{p=1}^{P} N_{p}$ ) and we have $N^{\prime}-N$ subcarriers that are always idle. The idle subcarriers are used to simplify the design of the reconstruction filter; they can also be employed to define unused regions of the spectrum (e.g., when the transmission band is fragmented [23]). Figure 1 illustrates the system in the uplink case.

\section{A. Downlink Transmission}

The OFDMA signal can be written as the sum of blocks generated at a rate $R_{B}=1 / T_{B}$, i.e. ${ }^{2}$

$$
s_{M C}(t)=\sum_{m} s_{m}\left(t-m T_{B}\right),
$$

where $s_{m}(t)$ is the signal associated to the $m$ th block. However, to simplify the notation we will ignore the dependence with the block number $m$, focusing on a single block.

The frequency-domain block transmitted on the downlink is $\left\{S_{k} ; k=0,1, \ldots, N^{\prime}-1\right\}$, where $S_{k}=\xi_{p} A_{k}^{(p)}$ for $k \in \Psi_{p}$, where the sets $\Psi_{p}$ and $\left\{A_{k}^{(p)} ; k \in \Psi_{p}\right\}$ are associated with the $p$ th user (it is assumed that $\Psi_{p} \cap \Psi_{p^{\prime}}=\emptyset$, i.e., different sets are assigned to different users) and $\xi_{p}$ is an appropriate weighting coefficient that accounts power control issues. The time-domain signal corresponding to that block is

$$
s(t)=\sum_{n} s_{n} w\left(\frac{n T}{N^{\prime}}\right) h_{T}\left(t-\frac{n T}{N^{\prime}}\right),
$$

where $T$ is the duration of the useful part of the block, $w(t)$ is a suitable time-domain window (used to reduce the out-of-band radiation levels [12]) and $h_{T}(t)$ is the impulse response of the reconstruction filter. Clearly, the duration of the block is the duration of $w(t)$. The block of time-domain samples $\left\{s_{n} ; n=0,1, \ldots, N^{\prime}-1\right\}$ is the IDFT (Inverse Discrete Fourier Transform) of the frequency-domain block $\left\{S_{k} ; k=0,1, \ldots, N^{\prime}-1\right\}$ (as usual, it is assumed that the time-domain and frequency-domain blocks are periodic, with period $N^{\prime}$, i.e., $S_{k+l N^{\prime}}=S_{k}$ and $s_{n+l N^{\prime}}=s_{n}$ for any integer l).

If $E\left[S_{k}\right]=0(E[\cdot]$ denotes 'ensemble average') and

$$
E\left[S_{k} S_{k^{\prime}}^{*}\right]= \begin{cases}G_{S, k}, & k=k^{\prime} \\ 0, & \text { otherwise }\end{cases}
$$

\footnotetext{
${ }^{2}$ Although the duration of each block can be smaller or larger than $T_{B}$, it is usually $T_{B}$.
}

then it can be shown that the PSD of the OFDMA signal is given $b y^{3}$

$$
G_{s}(f)=\frac{\left|H_{T}(f)\right|^{2}}{T^{2} T_{B}} \sum_{k=-N / 2}^{N / 2-1} G_{S, k}\left|W^{\text {eq }}\left(f-\frac{k}{T}\right)\right|^{2},
$$

where $H_{T}(f)=\mathcal{F}\left\{h_{T}(t)\right\}$ ( $\mathcal{F}$ denotes 'Fourier Transform') and

$$
W^{\mathrm{eq}}(f)=\sum_{l=-\infty}^{+\infty} W\left(f-\frac{l N^{\prime}}{T}\right),
$$

with $W(f)=\mathcal{F}\{w(t)\}$. When the number of used subcarriers is high ${ }^{4}\left(\sum_{p} N_{p}>>1\right)$ the time-domain coefficients $s_{n}$ can be approximately regarded as samples of a zero-mean complex Gaussian process. It can be easily demonstrated that $E\left[s_{n}\right]=0$ and that its autocorrelation is given by

$$
\begin{aligned}
R_{s, n-n^{\prime}} & =E\left[s_{n} s_{n^{\prime}}^{*}\right]= \\
& =\frac{1}{\left(N^{\prime}\right)^{2}} \sum_{k=-N / 2}^{N / 2-1} G_{S, k} \exp \left(j 2 \pi \frac{k\left(n-n^{\prime}\right)}{N^{\prime}}\right),
\end{aligned}
$$

i.e., the block $\left\{R_{s, n} ; n=0,1, \ldots, N^{\prime}-1\right\}$ is the IDFT of the block $\left\{\frac{1}{N^{\prime}} G_{S, k} ; k=0,1, \ldots, N^{\prime}-1\right\}$. Moreover

$$
R_{s, 0}=E\left[\left|s_{n}\right|^{2}\right]=2 \sigma^{2}=\frac{1}{\left(N^{\prime}\right)^{2}} \sum_{k=-N / 2}^{N / 2-1} G_{S, k},
$$

with $\sigma^{2}$ denoting the variance of the real and imaginary parts of $s_{n}$.

\section{B. Uplink Transmission}

For the uplink transmission we have a similar situation: the frequency-domain block to be transmitted by the $p$ th user is $\left\{S_{k}^{(p)} ; k=0,1, \ldots, N^{\prime}-1\right\}$, where

$$
S_{k}^{(p)}= \begin{cases}\xi_{p} A_{k}^{(p)}, & k \in \Psi_{p} \\ 0, & \text { otherwise }\end{cases}
$$

The corresponding time-domain signal is

$$
s^{(p)}(t)=\sum_{n} s_{n}^{(p)} w\left(\frac{n T}{N^{\prime}}\right) h_{T}\left(t-\frac{n T}{N^{\prime}}\right),
$$

where $\left\{s_{n}^{(p)} ; n=0,1, \ldots, N^{\prime}-1\right\}=\frac{1}{N^{\prime}}$ IDFT $\left\{S_{k}^{(p)} ; k=\right.$ $\left.0,1, \ldots, N^{\prime}-1\right\}$, with $E\left[s_{n}^{(p)}\right]=E\left[S_{k}^{(p)}\right]=0$ and

$$
E\left[S_{k}^{(p)} S_{k^{\prime}}^{(p) *}\right]= \begin{cases}G_{S, k}^{(p)}, & k=k^{\prime} \\ 0, & \text { otherwise }\end{cases}
$$

The PSD and autocorrelation of $s^{(p)}(t)$ are given by

$$
G_{s}^{(p)}(f)=\frac{\left|H_{T}(f)\right|^{2}}{T^{2} T_{B}} \sum_{k=-N / 2}^{N / 2-1} G_{S, k}^{(p)}\left|W^{\text {eq }}\left(f-\frac{k}{T}\right)\right|^{2}
$$

\footnotetext{
${ }^{3}$ We consider an even number of subcarriers.

${ }^{4}$ The number of active subcarriers can be a small fraction of $N$ for OFDMA signals, especially when the number of users is high.
} 
and

$$
\begin{aligned}
R_{s, n-n^{\prime}}^{(p)} & =E\left[s_{n}^{(p)} s_{n^{\prime}}^{(p) *}\right]= \\
& =\frac{1}{\left(N^{\prime}\right)^{2}} \sum_{k=-N / 2}^{N / 2-1} G_{S, k}^{(p)} \exp \left(j 2 \pi \frac{k\left(n-n^{\prime}\right)}{N^{\prime}}\right),
\end{aligned}
$$

respectively, with $\left\{R_{s, n}^{(p)} ; n=0,1, \ldots, N^{\prime}-1\right\}=\frac{1}{N^{\prime}}$ IDFT $\left\{G_{S, k}^{(p)} ; k=0,1, \ldots, N^{\prime}-1\right\}$ and

$$
R_{s, 0}^{(p)}=E\left[\left|s_{n}^{(p)}\right|^{2}\right]=2 \sigma_{p}^{2}=\frac{1}{\left(N^{\prime}\right)^{2}} \sum_{k=-N / 2}^{N / 2-1} G_{S, k}^{(p)},
$$

with $\sigma_{p}^{2}$ denoting the variance of the real and imaginary parts of $s_{n}^{(p)}$.

\section{Nonlinear EfFects on OFDMA Signals}

\section{A. Statistical Characterization of the Transmitted Signals}

We consider nonlinear signal processing schemes which operate on a sampled version of the OFDM signal. Moreover, these nonlinear operations are usually intentional (e.g., clipping and filtering techniques for reducing the PMEPR of the transmitted signals [12]). We will focus on the downlink transmission, but a similar analysis can be used for the uplink case. We just have to replace the samples $s_{n}$ by $s_{n}^{(p)}$, thus getting the characterization for the $P$ uplink transmitters.

The basic downlink transmitter structure considered in this section is depicted in Fig. 2. The nonlinear device $g_{C}(\cdot)$ is modeled as a bandpass memoryless nonlinearity operating on an oversampled version of the OFDMA signal, leading to

$$
s_{n}^{C}=g_{C}\left(\left|s_{n}\right|\right) \exp \left(j \arg \left(s_{n}\right)\right) .
$$

An optional frequency-domain filtering procedure, through the set of multiplying coefficients $\left\{F_{k} ; k=0,1, \ldots, N^{\prime}-1\right\}$, is considered to reduce the out-of-band radiation levels inherent to the nonlinear operation [12]. The transmitter structure of Fig. 2 is similar to the nonlinear signal processing schemes proposed in [12] for reducing the PMEPR of OFDM signals while maintaining the spectral efficiency of conventional OFDM schemes. For the uplink transmission, the set of coefficients $\left\{F_{k} ; k=0,1, \ldots, N^{\prime}-1\right\}$ can be used to eliminate the out-of-band radiation ${ }^{5}$ as well as the interference levels between users ${ }^{6}$, both introduced by the nonlinear device. For the downlink transmission the set of coefficients $\left\{F_{k} ; k=\right.$ $\left.0,1, \ldots, N^{\prime}-1\right\}$ can only be used to eliminate the out-ofband radiation, not the nonlinear interference between users.

We will take advantage of the Gaussian nature of OFDMA signals with a large number of subcarriers for the analytical characterization of the transmitted signals. It is well known that the output of a memoryless nonlinear device with a Gaussian input can be written as the sum of two uncorrelated

\footnotetext{
${ }^{5}$ Throughout this paper the "in-band" subcarriers are the $N$ subcarriers that can be assigned to users and the "out-of-band" subcarriers are the $N^{\prime}-N$ idle subcarriers associated to the oversampling.

${ }^{6}$ Due to the OFDMA nature of the signals, of the $N$ in-band subcarriers of the overall signal only $N_{p}$ are in fact in-band subcarriers of the $p$ th user. The remaining $N-N_{p}$ subcarriers are outside its in-band region.
}

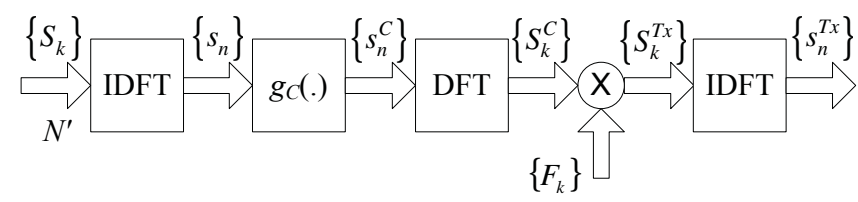

Fig. 2. Detail of the downlink transmitter structure with nonlinear signal processing.

components: a useful one, which is proportional to the input, and a self-interference one [24], i.e.,

$$
s_{n}^{C}=\alpha s_{n}+d_{n},
$$

where $E\left[s_{n} d_{n^{\prime}}^{*}\right]=0$ and

$$
\alpha=\frac{E\left[s_{n}^{C} s_{n}^{*}\right]}{E\left[\left|s_{n}\right|^{2}\right]}=\frac{E\left[R g_{C}(R)\right]}{E\left[R^{2}\right]}
$$

is given by (38), with $R=\left|s_{n}\right|$.

Using the results of the appendix, the autocorrelation of the output samples can be expressed as a function of the autocorrelation of the input samples, as follows

$$
R_{s, n-n^{\prime}}^{C}=E\left[s_{n}^{C} s_{n^{\prime}}^{C *}\right]=2 \sum_{\gamma=0}^{+\infty} P_{2 \gamma+1} f_{2 \gamma+1}^{R}\left(R_{s, n-n^{\prime}}\right),
$$

with $f_{2 \gamma+1}^{R}(\cdot)$ given by (40) and $P_{2 \gamma+1}$ denoting the total power associated to the Inter-Modulation Product (IMP) of order $2 \gamma+1$, which is given by (41). Since

$$
R_{s, n-n^{\prime}}^{C}=|\alpha|^{2} R_{s, n-n^{\prime}}+E\left[d_{n} d_{n^{\prime}}^{*}\right],
$$

it can be easily recognized that $P_{1}=|\alpha|^{2} \sigma^{2}$ and

$$
R_{d, n-n^{\prime}}=E\left[d_{n} d_{n^{\prime}}^{*}\right]=2 \sum_{\gamma=1}^{+\infty} P_{2 \gamma+1} f_{2 \gamma+1}^{R}\left(R_{s, n-n^{\prime}}\right) .
$$

It can be shown that

$$
E\left[S_{k}^{C} S_{k^{\prime}}^{C *}\right]= \begin{cases}N^{\prime} G_{S, k}^{C}, & k=k^{\prime} \\ 0, & \text { otherwise }\end{cases}
$$

with $\left\{G_{S, k}^{C} ; k=0,1, \ldots, N^{\prime}-1\right\}=$ DFT $\left\{R_{s, n}^{C} ; n=\right.$ $\left.0,1, \ldots, N^{\prime}-1\right\}$ and

$$
\begin{aligned}
G_{S, k}^{C} & =2 \sum_{\gamma=0}^{+\infty} \frac{P_{2 \gamma+1}}{\left(R_{s, 0}\right)^{2 \gamma+1}} f_{2 \gamma+1}^{G}\left(G_{S, k}\right)= \\
& =2 \sum_{\gamma=0}^{+\infty} \frac{P_{2 \gamma+1}}{\left(R_{s, 0}\right)^{2 \gamma+1}}\left(G_{S, k}\right)^{2 \gamma+1} .
\end{aligned}
$$

with $f_{2 \gamma+1}^{G}(\cdot)$ given by (45). Clearly

$$
G_{S, k}^{C}=|\alpha|^{2} G_{S, k}+G_{D, k},
$$

with

$$
G_{D, k}=2 \sum_{\gamma=1}^{+\infty} \frac{P_{2 \gamma+1}}{\left(R_{s, 0}\right)^{2 \gamma+1}}\left(G_{S, k}\right)^{2 \gamma+1},
$$

and $\left\{G_{D, k} ; k=0,1, \ldots, N^{\prime}-1\right\}=$ DFT $\left\{R_{d, n} ; n=\right.$ $\left.0,1, \ldots, N^{\prime}-1\right\}$. The average power of the signal at the nonlinearity output, $P_{\text {out }}$, can be obtained from (48), with useful and self-interference components $S=|\alpha|^{2} \sigma^{2}$ and $I=P_{\text {out }}-S=\sum_{\gamma=1}^{+\infty} P_{2 \gamma+1}$, respectively. 
Having in mind (14) and the signal processing chain in Fig. 2, the frequency-domain block $\left\{S_{k}^{T x}=S_{k}^{C} F_{k} ; k=\right.$ $\left.0,1, \ldots, N^{\prime}-1\right\}$ can obviously be decomposed into useful and nonlinear self-interference components:

$$
S_{k}^{T x}=\alpha S_{k} F_{k}+D_{k} F_{k}
$$

where $\left\{D_{k} ; k=0,1, \ldots, N^{\prime}-1\right\}=\operatorname{DFT}\left\{d_{n} ; n=\right.$ $\left.0,1, \ldots, N^{\prime}-1\right\}$. Clearly, $E\left[D_{k}\right]=0$ and

$$
E\left[D_{k} D_{k^{\prime}}^{*}\right]= \begin{cases}N^{\prime} G_{D, k}, & k=k^{\prime} \\ 0, & \text { otherwise }\end{cases}
$$

Moreover, $D_{k}$ exhibits quasi-Gaussian characteristics for any $k$, provided that the number of subcarriers is high enough. Therefore, $E\left[S_{k}^{T x} S_{k^{\prime}}^{T x *}\right]=0$ for $k \neq k^{\prime}$, and $E\left[\left|S_{k}^{T x}\right|^{2}\right]=$ $\left|F_{k}\right|^{2} E\left[\left|S_{k}^{C}\right|^{2}\right]=N^{\prime}\left|F_{k}\right|^{2} G_{S, k}^{C}$.

\section{B. Signal to Interference Ratio Levels on the Received Signals}

1) Multiple Users: Let us consider the transmission of the OFDMA downlink signal over a frequency-selective channel. It is clear that the received symbol on the $k$ th subcarrier is

$$
Y_{k}^{(p)}=\alpha \xi_{p} F_{k} H_{k}^{(p)} A_{k}^{(p)}+F_{k} H_{k}^{(p)} D_{k}+N_{k}^{(p)},
$$

with $H_{k}^{(p)}$ and $N_{k}^{(p)}$ denoting the corresponding channel frequency response and channel noise, respectively. We can calculate a signal-to-interference ratio (SIR) on each subcarrier, given by

$$
\operatorname{SIR}_{k}^{(p)}=\frac{E\left[\left|\alpha \xi_{p} A_{k}^{(p)}\right|^{2}\right]}{E\left[\left|D_{k}\right|^{2}\right]}
$$

and also an equivalent signal to noise plus self-interference ratio (ESNR) on each subcarrier, given by

$$
\operatorname{ESNR}_{k}^{(p)}=\frac{\left|F_{k}\right|^{2}\left|H_{k}^{(p)}\right|^{2} E\left[\left|\alpha \xi_{p} A_{k}^{(p)}\right|^{2}\right]}{\left|F_{k}\right|^{2}\left|H_{k}^{(p)}\right|^{2} E\left[\left|D_{k}\right|^{2}\right]+E\left[\left|N_{k}^{(p)}\right|^{2}\right]} .
$$

When transmitting an OFDMA uplink signal over a frequency-selective channel, the received symbol on the $k$ th subcarrier of user $p$ is

$$
Y_{k}^{(p)}=\alpha_{p} \xi_{p} F_{k}^{(p)} H_{k}^{(p)} A_{k}^{(p)}+\sum_{p^{\prime}=1}^{P} F_{k}^{\left(p^{\prime}\right)} H_{k}^{\left(p^{\prime}\right)} D_{k}^{\left(p^{\prime}\right)}+N_{k} .
$$

In this case $\operatorname{SIR}_{k}^{(p)}$ is given by

$$
\operatorname{SIR}_{k}^{(p)}=\frac{\left|F_{k}^{(p)}\right|^{2}\left|H_{k}^{(p)}\right|^{2} E\left[\left|\alpha_{p} \xi_{p} A_{k}^{(p)}\right|^{2}\right]}{I_{k}^{\mathrm{eq}}},
$$

with

$$
I_{k}^{\mathrm{eq}}=\sum_{p^{\prime}=1}^{P}\left|F_{k}^{\left(p^{\prime}\right)}\right|^{2}\left|H_{k}^{\left(p^{\prime}\right)}\right|^{2} E\left[\left|D_{k}^{\left(p^{\prime}\right)}\right|^{2}\right],
$$

and $\operatorname{ESNR}_{k}^{(p)}$ is given by

$$
\operatorname{ESNR}_{k}^{(p)}=\frac{\left|F_{k}^{(p)}\right|^{2}\left|H_{k}^{(p)}\right|^{2} E\left[\left|\alpha_{p} \xi_{p} A_{k}^{(p)}\right|^{2}\right]}{I_{k}^{\mathrm{eq}}+E\left[\left|N_{k}\right|^{2}\right]} .
$$

2) Single User: When we have just one active user, e.g. the $p$ th user, and, on the downlink case, we have $Y_{k}^{(p)}=$ $\alpha \xi_{p} F_{k} H_{k}^{(p)} A_{k}^{(p)}+F_{k} H_{k}^{(p)} D_{k}+N_{k}$ which gives

$$
\operatorname{SIR}_{k}^{(p)}=\frac{E\left[\left|\alpha \xi_{p} A_{k}^{(p)}\right|^{2}\right]}{E\left[\left|D_{k}\right|^{2}\right]}
$$

and

$$
\operatorname{ESNR}_{k}^{(p)}=\frac{\left|F_{k}\right|^{2}\left|H_{k}^{(p)}\right|^{2} E\left[\left|\alpha \xi_{p} A_{k}^{(p)}\right|^{2}\right]}{\left|F_{k}\right|^{2}\left|H_{k}^{(p)}\right|^{2} E\left[\left|D_{k}\right|^{2}\right]+E\left[\left|N_{k}\right|^{2}\right]} .
$$

For the uplink we have a similar situation, with $Y_{k}^{(p)}=$ $\alpha_{p} \xi_{p} F_{k}^{(p)} H_{k}^{(p)} A_{k}^{(p)}+F_{k}^{(p)} H_{k}^{(p)} D_{k}^{(p)}+N_{k}$, for $k \in \Psi_{p}$ and zero elsewhere. This means that

$$
\operatorname{SIR}_{k}^{(p)}=\frac{E\left[\left|\alpha_{p} \xi_{p} A_{k}^{(p)}\right|^{2}\right]}{E\left[\left|D_{k}^{(p)}\right|^{2}\right]}
$$

and

$$
\operatorname{ESNR}_{k}^{(p)}=\frac{\left|F_{k}^{(p)}\right|^{2}\left|H_{k}^{(p)}\right|^{2} E\left[\left|\alpha_{p} \xi_{p} A_{k}^{(p)}\right|^{2}\right]}{\left|F_{k}^{(p)}\right|^{2}\left|H_{k}^{(p)}\right|^{2} E\left[\left|D_{k}^{(p)}\right|^{2}\right]+E\left[\left|N_{k}\right|^{2}\right]} .
$$

\section{Performance Results}

In this section we present a set of results concerning the performance evaluation of the proposed nonlinear signal processing schemes that operate on a sampled version of the OFDMA signal. The considered OFDMA system has $N=256$ subcarriers and a QPSK (Quadrature Phase-Shift Keying) constellation, with a Gray mapping rule, on each subcarrier. The system has $P=4$ users, not necessarily active, with an equal number of subcarriers assigned to each user, i.e., $N_{p}=64, p=1,2,3,4$. We consider an ideal envelope clipping, with clipping level $s_{M} / \sigma=2.0$, unless otherwise stated. With this clipping level the PMEPR exceeded only $0.1 \%$ of the time (which is almost independent of the number of used subcarriers [12]) is about $5.7 \mathrm{~dB}$ when the $F_{k}$ coefficients are used to remove the nonlinear distortion in the unused subcarriers; for $F_{k}=1$ (no post-clipping filtering) the PMEPR is about $3.5 \mathrm{~dB}$ and without clipping the PMEPR is about $8.4 \mathrm{~dB}$. Unless otherwise stated, the oversampling factor is $M_{T x}=N^{\prime} / N=2$ and the multiplying coefficients $\left\{F_{k} ; k=0,1, \ldots, N^{\prime}-1\right\}$ and $\left\{F_{k}^{(p)} ; k=0,1, \ldots, N^{\prime}-1\right\}$ are set to 1 for the used subcarriers and 0 for the unused and out-of-band subcarriers.

\section{A. Downlink Transmission}

In the case of downlink transmission, we consider two different ways of assigning subcarriers to a given user: regular grid (RG) or block assignment (BA), as show in Fig. 3. Regular grid has the advantage of having higher multipath diversity. This is a consequence of not using adjacent subcarriers for the same user, which leads to less correlated channel coefficients. A RG is not suitable for the uplink since it requires perfect carrier synchronization between users (for BA we can employ idle subcarriers between blocks to reduce the degradation due to carrier synchronization errors between users).

In Fig. 4 we compare the theoretical values of $E\left[\left|D_{k}\right|^{2}\right]$, measured in $\mathrm{dB}$, obtained using the analytical approach presented in this paper, with the simulated ones. We consider the downlink transmission and different cases concerning the way the power is attributed to the users. The figure clearly 


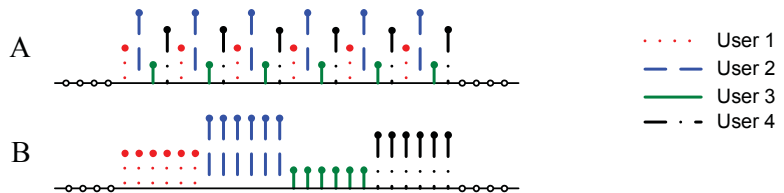

Fig. 3. User subcarrier assignment: regular grid (A) and block assignment (B).

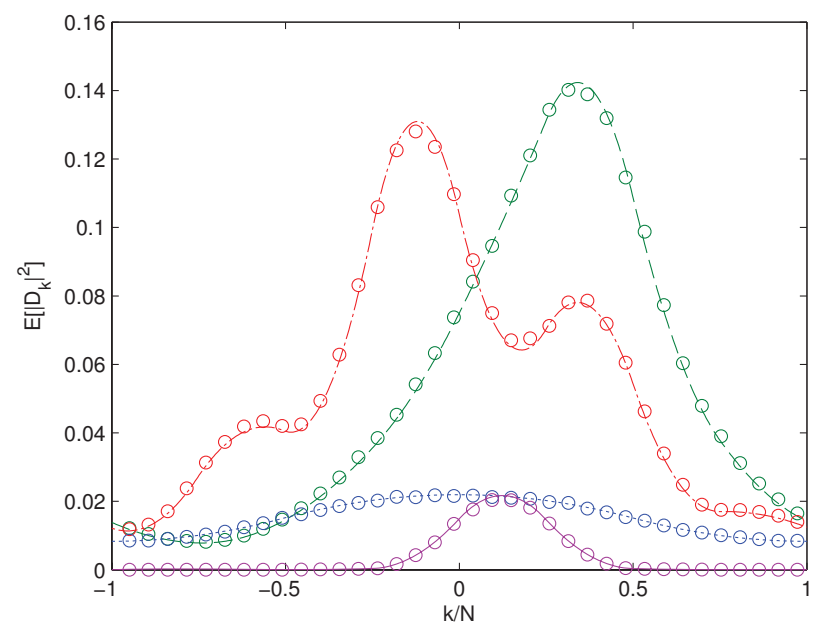

Fig. 4. Comparison of simulated (o) and analytical results for downlink transmission with four equal power $0 \mathrm{~dB}$ users (dotted line), just one $0 \mathrm{~dB}$ user active (solid line) and BA with $\left\{\xi_{1}, \xi_{2}, \xi_{3}, \xi_{4}\right\}=\{-10,20,0,10\} \mathrm{dB}$ (dash-dotted line) or $\left\{\xi_{1}, \xi_{2}, \xi_{3}, \xi_{4}\right\}=\{-10,0,10,20\} \mathrm{dB}$ (dashed line).

shows a good match between theory and simulation, even when we have only one user (64 active subcarriers). Naturally, the accuracy of our analytical approach decreases when the number of active subcarriers is very low.

A comparison of results obtained for self-interference values with and without the use of oversampling can be found in Fig. 5 for different number of users (i.e, different systems loads), with equal power users and block assignment. It can be seen that we can improve the SIR levels for a fully loaded system (all users active) and two users active by employing an oversampling factor of 2 (negligible improvements are observed with an oversampling factor of 4). If we have just one user active oversampling is not necessary (i.e., the SIR levels are almost independent of the oversampling factor when we have a single user). Total power of the nonlinear distortion component is independent of the oversampling factor (it is only a function of the normalized clipping level), but part of it is outside the used part of the spectrum when we have oversampling (see also [12]); the same applies when the system is not fully loaded. The increase in the values of $E\left[\left|D_{k}\right|^{2}\right]$ at the edge of the spectrum ( $N^{\prime}$ subcarriers) observed in (B) and (C) for $M_{T x}=1$ and $M_{T x}=2$ is due to the aliasing effect. For just one user the values of $E\left[\left|D_{k}\right|^{2}\right]$ are similar to the ones in the uplink case. Fig. 6 shows the impact of the normalized clipping level on $E\left[\left|\operatorname{SIR}_{k}^{(p)}\right|^{2}\right]$ with equal power users for different system loads and oversampling factors. It can be seen that the improvement obtained by using an oversampling factor of 2 is independent of the clipping level. We can notice again that when there is just one user active, the SIR levels are almost independent of oversampling.
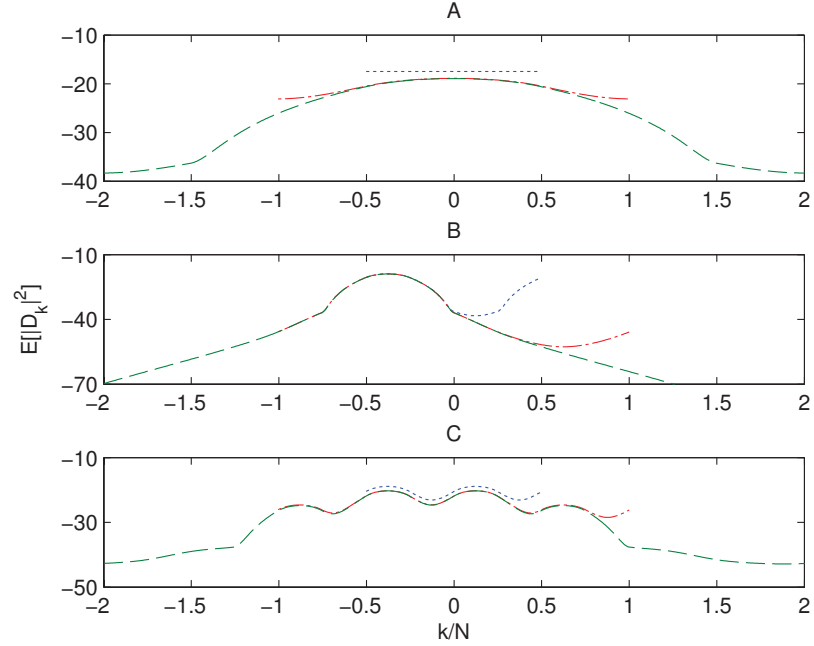

Fig. 5. Comparison of $E\left[\left|D_{k}\right|^{2}\right]$ for downlink transmission with equal power users and BA with all users active (A), user 1 active (B) and users 1 and 3 active (C), for $M_{T x}=1$ (dotted line), $M_{T x}=2$ (dash-dotted line) and $M_{T x}=4$ (dashed line).
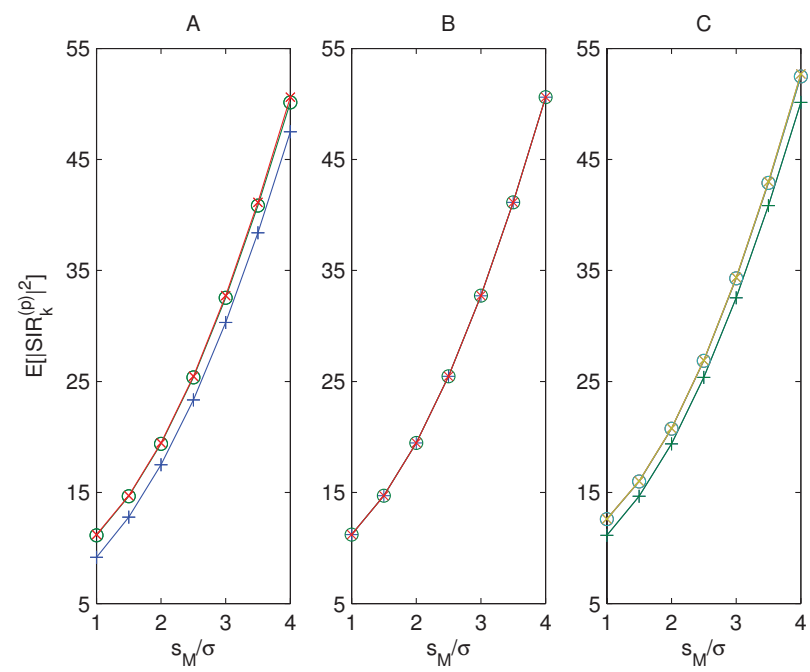

Fig. 6. Variation of $E\left[\left|\operatorname{SIR}_{k}^{(p)}\right|^{2}\right]$ for downlink transmission and BA with equal power users with all users active (A), user 1 active (B) and users 1 and 3 active (C), for $M_{T x}=1(+), M_{T x}=2(\circ)$ and $M_{T x}=4(\times)$.

Fig. 7 compares the variation of $E\left[\left|\alpha \xi_{p} A_{k}^{(p)}\right|^{2}\right]$ and $E\left[\left|D_{k}\right|^{2}\right]$ for block assignment and the two different user attribution of the power control coefficients $\{-10,0,10,20\}$ $\mathrm{dB}$ used before. From the figure it is clear that increasing power of the users within the band leads to significantly lower levels of self-interference and that low-power users can suffer high levels of interference when they are between users with higher attributed powers. Figs. 8 to 10 concern the comparison of two subcarrier assigning schemes: RG and BA. Fig. 8 compares $E\left[\left|\operatorname{SIR}_{k}^{(p)}\right|^{2}\right]$ for the two schemes and different power users. The SIR levels shown concern the $-10 \mathrm{~dB}$-power and the $0 \mathrm{~dB}$-power users. Equal power users and the two different user attribution of the power control coefficients $\{-10,0,10,20\} \mathrm{dB}$ used before are considered. Figs. 9 and 10 show the impact of subcarrier assignment on $\operatorname{SIR}_{k}^{(p)}$ and 

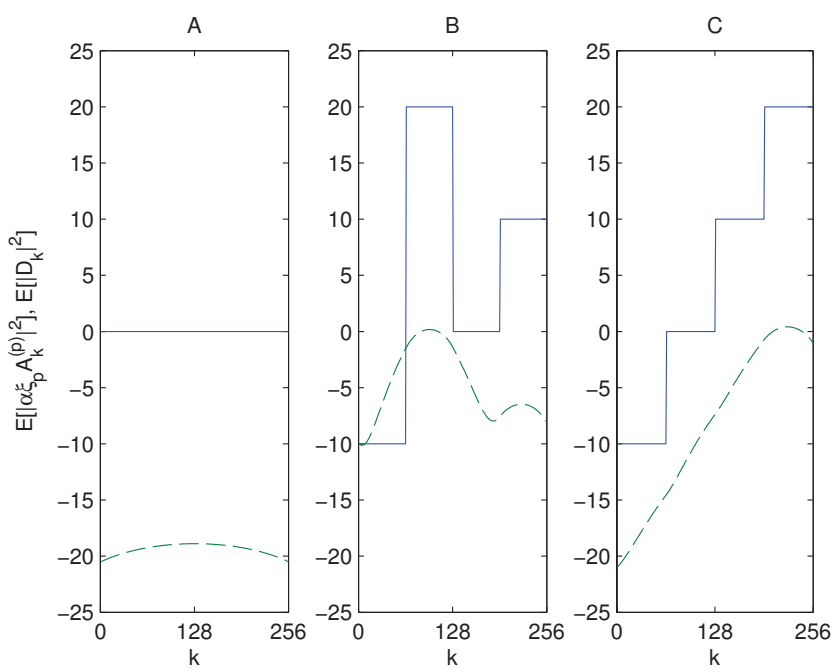

Fig. 7. Variation of $E\left[\left|\alpha \xi_{p} A_{k}^{(p)}\right|^{2}\right]$ (solid line) and $E\left[\left|D_{k}\right|^{2}\right]$ (dashed line) for downlink transmission and $\mathrm{BA}$ with equal $0 \mathrm{~dB}$ power users (A), $\left\{\xi_{1}, \xi_{2}, \xi_{3}, \xi_{4}\right\}=\{-10,20,0,10\} \mathrm{dB}$ (B) and $\left\{\xi_{1}, \xi_{2}, \xi_{3}, \xi_{4}\right\}=$ $\{-10,0,10,20\} \mathrm{dB}(\mathrm{C})$.
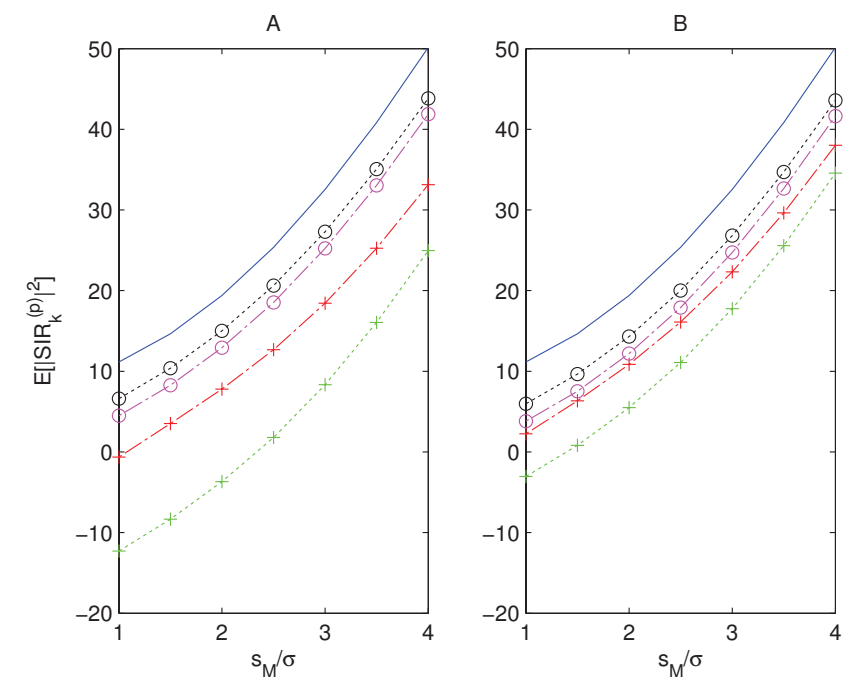

Fig. 8. Variation of $E\left[\left|\operatorname{SIR}_{k}^{(p)}\right|^{2}\right]$ for downlink transmission with equal power $0 \mathrm{~dB}$ users (solid line) and RG (o) or BA (+), with $\left\{\xi_{1}, \xi_{2}, \xi_{3}, \xi_{4}\right\}=$ $\{-10,20,0,10\} \mathrm{dB}$ (dotted line) or $\left\{\xi_{1}, \xi_{2}, \xi_{3}, \xi_{4}\right\}=\{-10,0,10,20\} \mathrm{dB}$ (dash-dotted line), for the user with $\xi_{p}=-10 \mathrm{~dB}(\mathrm{~A})$ and $\xi_{p}=0 \mathrm{~dB}$ (B).

$E\left[\left|D_{k}\right|^{2}\right]$, respectively. Again, we consider two different user attribution of the same power control coefficients. Clearly, the BA scheme allows better SIR levels for users with lower power, although slightly worse for users with higher power. As in Fig. 7, it can be seen that the performance of low-power users can be severely affected when they are between users with higher attributed powers.

\section{B. Uplink Transmission}

In the uplink transmission post-clipping filtering is generally used to eliminate the out-of-band radiation. Fig. 11 compares the variation of $\operatorname{SIR}_{k}^{(p)}$ for different attribution of the power control coefficients $\{-10,0,10,20\} \mathrm{dB}$ in case no postclipping filtering is used. Again these coefficients are assigned
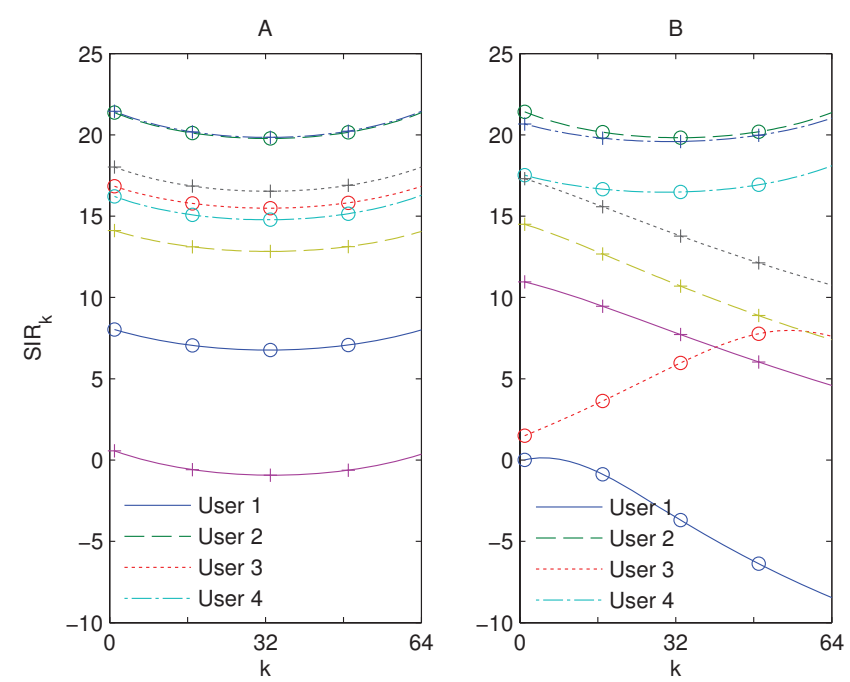

Fig. 9. Comparison of $\operatorname{SIR}_{k}^{(p)}$ for downlink transmission with $\mathrm{RG}(\mathrm{A})$ and BA (B) with $\left\{\xi_{1}, \xi_{2}, \xi_{3}, \xi_{4}\right\} \stackrel{k}{=}\{-10,20,0,10\} \mathrm{dB}$ (o) or $\left\{\xi_{1}, \xi_{2}, \xi_{3}, \xi_{4}\right\}=$ $\{-10,0,10,20\} \mathrm{dB}(+)$.
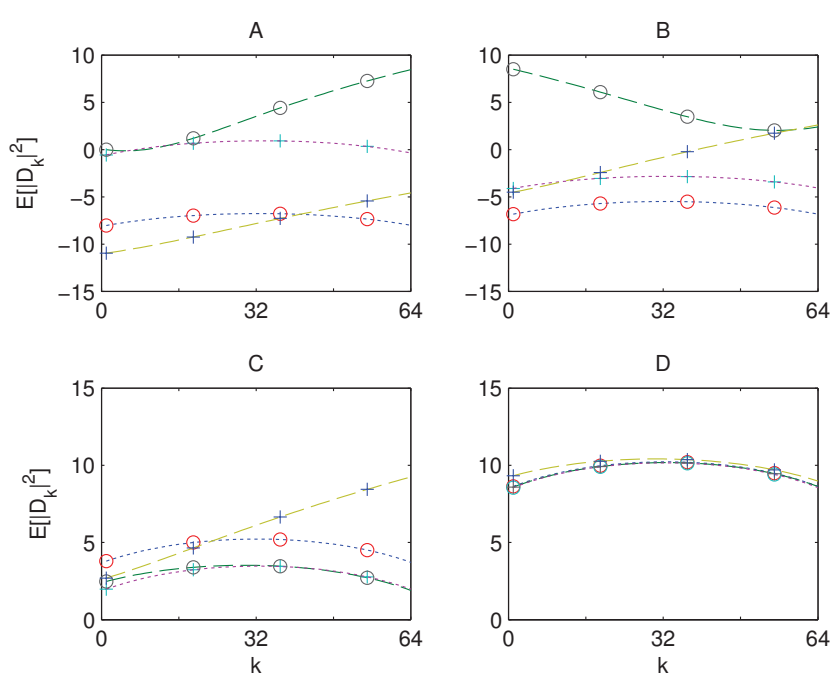

Fig. 10. Comparison of $E\left[\left|D_{k}\right|^{2}\right]$ for downlink transmission with RG (dotted line) and BA (dashed line), with $\left\{\xi_{1}, \xi_{2}, \xi_{3}, \xi_{4}\right\}=\{-10,20,0,10\} \mathrm{dB}$ (०) or $\left\{\xi_{1}, \xi_{2}, \xi_{3}, \xi_{4}\right\}=\{-10,0,10,20\} \mathrm{dB}(+)$, for the user with $\xi_{p}=-10$ $\mathrm{dB}(\mathrm{A}), \xi_{p}=0 \mathrm{~dB}(\mathrm{~B}), \xi_{p}=10 \mathrm{~dB}(\mathrm{C})$ and $\xi_{p}=20 \mathrm{~dB}(\mathrm{D})$.

in two different ways: no particular order or increasing order. Clearly, we have a behavior similar to the one observed for the downlink transmission, namely worse SIR levels for lowpower users, especially when they use sets of subcarriers that are adjacent to high-power subcarriers (i.e., when we have low-power users close to high-power users). Naturally, if we have a post-clipping filtering able to eliminate the nonlinear distortion for the subcarriers that are not used to a given user then the SIR levels of a given user are only a function of the clipping for that user, even when we have low-power users adjacent to high-power users. Therefore, in contrast to the downlink case, the post-clipping filtering can increase the system's robustness significantly.

Let us now consider the transmission over a frequencyselective channel. Fig. 12 shows $\operatorname{ESNR}_{k}^{(p)}$ for the $0 \mathrm{~dB}$-power 

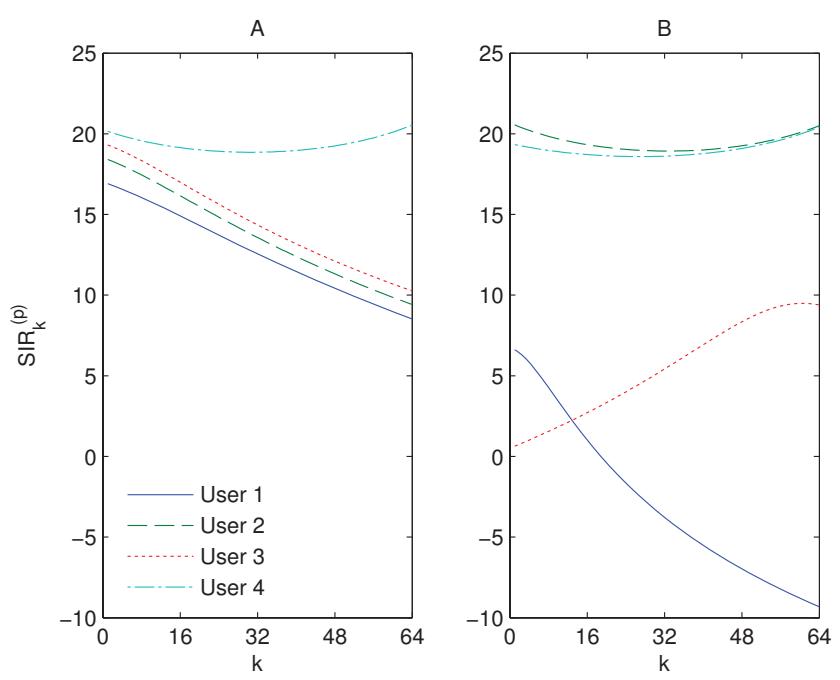

Fig. 11. Comparison of $\operatorname{SIR}_{k}^{(p)}$ for uplink transmission without postclipping filtering with $\left\{\xi_{1}, \xi_{2}, \xi_{3}, \xi_{4}\right\}=\{-10,0,10,20\} \mathrm{dB}$ (A) and $\left\{\xi_{1}, \xi_{2}, \xi_{3}, \xi_{4}\right\}=\{-10,20,0,10\} \mathrm{dB}(\mathrm{B})$.
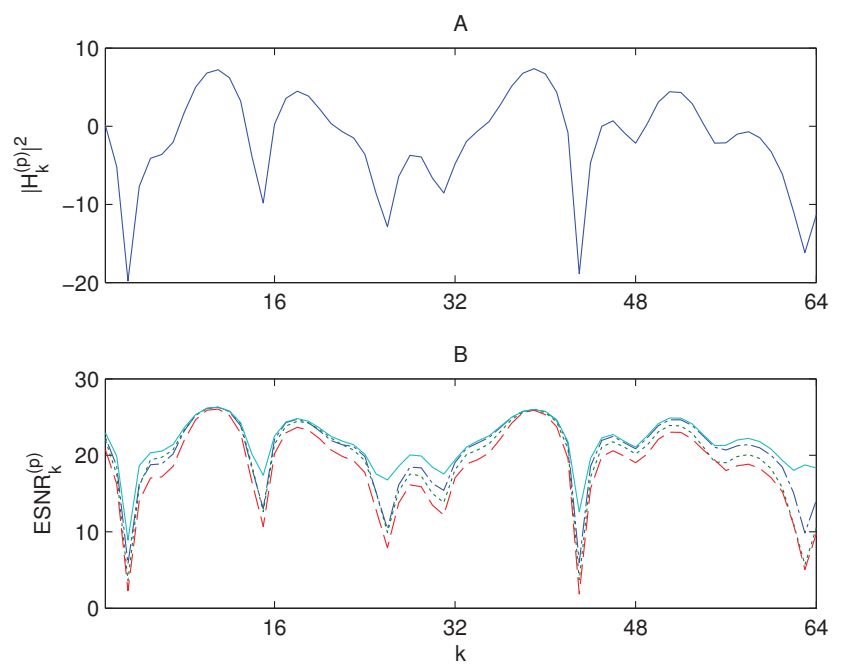

Fig. 12. Variation of $\left|H_{k}^{(p)}\right|^{2}$ (A) and comparison of $\operatorname{ESNR}_{k}^{(p)}$ (B) for uplink transmission with post-clipping filtering (solid line) and without post-clipping filtering and equal power $0 \mathrm{~dB}$ users (dash-dotted line), $\left\{\xi_{1}, \xi_{2}, \xi_{3}, \xi_{4}\right\}=$ $\{-10,20,0,10\} \mathrm{dB}$ (dashed line) or $\left\{\xi_{1}, \xi_{2}, \xi_{3}, \xi_{4}\right\}=\{-10,0,10,20\}$ $\mathrm{dB}$ (dotted line), for the user with $\xi_{p}=0 \mathrm{~dB}$.

user with and without post-clipping filtering for different attribution of power control coefficients. The results for 4 equal-power users (each with $0 \mathrm{~dB}$ ) are also included for the sake of comparison. From this figure it is clear that the ESNR values are much better when we have post-clipping filtering, especially for the subcarriers that have deep fading. This is aggravated for low-power users, especially when they are adjacent to high-power users.

\section{Conclusions}

In this paper we presented an analytical tool to evaluate nonlinear distortion effects on systems employing OFDMA signals. Our results allow an analytical spectral characterization of the transmitted signals, as well as the computation of the nonlinear interference levels on the received signals. They can also be used to find the corresponding BER. A set of performance results was presented, showing that the power allocated to each user has a key impact on the nonlinear distortion effects. Users with smaller allocated power face stronger interference levels and increasing attribution of power control coefficients can lead to lower interference levels. It is also shown that nonlinear distortion levels are significantly different when just a small fraction of the subcarriers is used (i.e., when the system load is small).

\section{APPENDIX}

Let us consider a Gaussian signal whose complex envelope $x(t)$ has zero mean and autocorrelation $R_{x}(\tau)$. If this signal is submitted to a bandpass memoryless nonlinearity then the complex envelope of the signal at the power amplifier output can be written as

$$
y(t)=g(|x(t)|) \exp (j \arg (x(t))) .
$$

It can be shown [24] that $y(t)$ can be decomposed into uncorrelated useful and self-interference components, as follows:

$$
y(t)=\alpha x(t)+d(t)
$$

where $E\left[y(t) x(t-\tau)^{*}\right]=0$ and

$$
\begin{aligned}
\alpha & =\frac{E\left[y(t) x(t)^{*}\right]}{E\left[|x(t)|^{2}\right]}=\frac{E[R g(R)]}{E\left[R^{2}\right]}= \\
& =\frac{1}{2 \sigma^{2}} \int_{0}^{+\infty} R A(R) \exp (j \Theta(R)) \frac{R}{\sigma^{2}} \exp \left(-\frac{R^{2}}{2 \sigma^{2}}\right) d R,
\end{aligned}
$$

with $\sigma^{2}=E\left[|x(t)|^{2}\right] / 2, R=|x(t)|, A(R)=|g(R)|$ and $\Theta(R)=\arg (g(R))$.

It can also be shown [24] that the autocorrelation of the output samples can be expressed as a function of the autocorrelation of the input samples, as follows

$$
R_{y}(\tau)=E\left[y(t) y(t-\tau)^{*}\right]=2 \sum_{\gamma=0}^{+\infty} P_{2 \gamma+1} f_{2 \gamma+1}^{R}\left(R_{x}(\tau)\right)
$$

with $f_{2 \gamma+1}^{R}(\cdot)$ given by

$$
f_{2 \gamma+1}^{R}(R(\tau)) \triangleq \frac{(R(\tau))^{\gamma+1}\left(R^{*}(\tau)\right)^{\gamma}}{(R(0))^{2 \gamma+1}}
$$

and $P_{2 \gamma+1}$ denoting the total power associated to the InterModulation Product (IMP) of order $2 \gamma+1$, which can be obtained from [25]:

$$
P_{2 \gamma+1}=\frac{\left|v_{2 \gamma+1}\right|^{2}}{2 \gamma !(\gamma+1) !}
$$

where

$$
v_{2 \gamma+1}=\int_{0}^{+\infty} R g_{C}(R) W_{2 \gamma+1}\left(\frac{R}{\sqrt{2 \sigma^{2}}}\right)
$$

with

$$
W_{2 \gamma+1}(x)=\frac{\gamma !}{2} x \exp \left(-x^{2}\right) L_{\gamma}^{(1)}\left(x^{2}\right),
$$

and $L_{\gamma}^{(1)}(\cdot)$ denoting a generalized Laguerre polynomial of order $\gamma[26]$. 
Therefore, the PSD of $y(t)$ is

$$
G_{y}(f)=\mathcal{F}\left\{R_{y}(\tau)\right\}=2 \sum_{\gamma=0}^{+\infty} \frac{P_{2 \gamma+1}}{\left(R_{x}(0)\right)^{2 \gamma+1}} f_{2 \gamma+1}^{G}\left(G_{x}(f)\right),
$$

where

$$
f_{2 \gamma+1}^{G}(G(f)) \triangleq \underbrace{G(-f) * \ldots * G(-f)}_{\gamma} * \underbrace{G(f) * \ldots * G(f)}_{\gamma+1} .
$$

Clearly $R_{y}(\tau)=|\alpha|^{2} R_{x}(\tau)+R_{d}(\tau)$, with the autocorrelation of the self-interference component given by

$$
R_{d}(\tau)=E\left[d(t) d(t-\tau)^{*}\right]=2 \sum_{\gamma=1}^{+\infty} P_{2 \gamma+1} f_{2 \gamma+1}^{R}\left(R_{x}(\tau)\right)
$$

and $G_{y}(f)=|\alpha|^{2} G_{x}(f)+G_{d}(f)$, with

$$
G_{d}(f)=\mathcal{F}\left\{R_{y}(\tau)\right\}=2 \sum_{\gamma=1}^{+\infty} \frac{P_{2 \gamma+1}}{\left(R_{x}(0)\right)^{2 \gamma+1}} f_{2 \gamma+1}^{G}\left(G_{x}(f)\right) .
$$

The average power of the signal at the nonlinearity output is given by

$$
P_{\text {out }}=\frac{1}{2} E\left[g^{2}(R)\right]=\frac{1}{2} \int_{0}^{+\infty} g^{2}(R) \frac{R}{\sigma^{2}} \exp \left(-\frac{R^{2}}{2 \sigma^{2}}\right) d R,
$$

the average power of the useful component is $S=|\alpha|^{2} \sigma^{2}$ and the average power of the self-interference component is

$$
I=P_{\text {out }}-S=\frac{1}{2} R_{d}(0)=\sum_{\gamma=1}^{+\infty} P_{2 \gamma+1}
$$

\section{REFERENCES}

[1] H. Sari, Y. Levy, and G. Karam, "An analysis of orthogonal frequencydivision multiple access," in Proc. IEEE Global Telecommun. Conf. (GLOBECOM'97), Phoenix, AZ, USA, Nov. 1997.

[2] I. Koffman and V. Roman, "Broadband wireless access solutions based on OFDM access in IEEE 802.16," IEEE Commun. Mag., vol. 40, no. 4, pp. 96-103, Apr. 2002.

[3] L. Cimini, "Analysis and simulation of a digital mobile channel using orthogonal frequency division multiplexing," IEEE Trans. Commun., vol. 33, no. 7, pp. 665-675, July 1985.

[4] J. Bingham, "Multicarrier modulation for data transmission: an idea whose time has come," IEEE Commun. Mag., vol. 28, no. 5, pp. 5-14, May 1990.

[5] IEEE Standard for Local and Metropolitan Area Networks - Part 16: Air Interface for Fixed Broadband Wireless Access Systems, IEEE 802.162004, Oct. 2004.

[6] 3rd Generation Partnership Project: Technical Specification Group Radio Access Network; Physical Layers Aspects for Evolved UTRA, 3GPPP TR 25.814, 2006.

[7] IEEE 802.22 Working Group on Wireless Regional Area Networks. [Online]. Available: http://www.ieee802.org/22/

[8] Part 16: Air Interface for Fixed and Mobile Broadband Wireless Access Systems Amendment 2: Physical and Medium Access Control Layers for Combined Fixed and Mobile Operation in Licensed Bands and Corrigendum 1, IEEE 802.16-2005, Feb. 2006.

[9] D. Galda and H. Rohling, "A low-complexity transmitter structure for OFDM-FDMA uplink systems," in Proc. IEEE Veh. Technol. Conf. (VTC'02 Spring), Birmingham, AL, USA, May 2002, pp. 1737-1741.

[10] P. Xia, S. Zhou, and G. Giannakis, "Bandwidth- and power-efficient multi-carrier multiple access," in Proc. IEEE Wireless Commun. Netw. Conf. (WCNC'03), New Orleans, LA, USA, Mar. 2003, pp. 207-211.

[11] R. Dinis, D. Falconer, C. Lam, and M. Sabbaghian, "A multiple access scheme for the uplink of broadband wireless systems," in Proc. IEEE 47th Global Telecommun. Conf. (GLOBECOM'04), Dallas, TX, USA, Nov./Dec. 2004.
[12] R. Dinis and A. Gusmão, "A class of nonlinear signal processing schemes for bandwidth-efficient OFDM transmission with low envelope fluctuation," IEEE Trans. Commun., vol. 52, no. 11, pp. 2009-2018, Nov. 2004.

[13] X. Li and L. Cimini, "Effects of clipping and filtering on the performance of OFDM," IEEE Commun. Lett., vol. 2, no. 5, May 1998.

[14] J. Armstrong, "Peak-to-average power reduction for OFDM by repeated clipping and frequency-domain filtering," IEE Electron. Lett., vol. 38, no. 5, pp. 246-247, Feb. 2002.

[15] R. Dinis and P. Silva, "Analytical evaluation of nonlinear effects in MC-CDMA signals," IEEE Trans. Wireless Commun., vol. 5, no. 8, pp. 2277-2284, Aug. 2006

[16] G. Santella and F. Mazzenga, "A model for performance evaluation in M-QAM-OFDM schemes in presence of nonlinear distortions," in Proc. IEEE 45th Veh. Technol. Conf. (VTC'05 Spring), vol. 2, Chicago, USA, July 1995 , pp. 830-834.

[17] P. Banelli and S. Cacopardi, "Theoretical analysis and performance of OFDM signals in nonlinear AWGN channels," IEEE Trans. Commun., vol. 48, no. 3, pp. 430-441, Mar. 2000.

[18] D. Dardari, "Exact analysis of joint clipping and quantization effects in high speed WLAN receivers," in Proc. IEEE International Conf. Commun. (ICC'03), vol. 5, Ankorage, Canada, May 2003, pp. 34873492.

[19] - "Joint clip and quantization effects characterization in OFDM receivers," IEEE Trans. Circuits Syst. I, vol. 53, no. 8, pp. 1741-1748, Aug. 2006.

[20] T. Araújo and R. Dinis, "Performance evaluation of quantization effects on multicarrier modulated signals," IEEE Trans. Veh. Technol., vol. 56, no. 5, pp. 2922-2930, Sep. 2007.

[21] A. Saleh, "Frequency-independent and frequency-dependent nonlinear models of TWT amplifiers," IEEE Trans. Commun., vol. 29, no. 11, pp. 1715-1720, Nov. 1981.

[22] D. Dardari, V. Tralli, and A. Vaccari, "A theoretical characterization of nonlinear distortion effects in OFDM systems," IEEE Trans. Commun., vol. 48 , no. 10 , pp. $1755-1764$, Oct. 2000.

[23] E. Dahlman, P. Frenger, J. Guey, G. Klang, R. Ludwig, M. Meyer, N. Wiberg, and K. Zangi, "A framework for future radio access," in Proc. IEEE 61th Veh. Technol. Conf. (VTC'05 Spring), vol. 5, Phoenix, AZ, USA, May/June 2005, pp. 2944-2948.

[24] H. Rowe, "Memoryless nonlinearities with Gaussian input: elementary results," Bell System Tech. J., vol. 61, Sep. 1982.

[25] G. Stette, "Calculation of intermodulation from a single carrier amplitude characteristic," IEEE Trans. Commun., vol. 22, no. 3, pp. 319-323, Mar. 1974.

[26] M. Abramowitz and I. Stegun, Handbook of Mathematical Functions. New York: Dover Publications, 1972.

Teresa Araújo received a degree in Applied Maths/Computer Science from University of Porto, Porto, Portugal and a MSc in Electrical and Computer Engineering from Instituto Superior Técnico (IST), Technical University of Lisbon, Portugal, in 1991 and 2004, respectively. She is a teacher at the Math Department from Instituto Superior de Engenharia do Porto (ISEP) since 1993. From 2006 to 2008 she did research at the Signal and Image Processing Group from Institute of Systems and Robotics (ISR/IST) and in 2009 she joined the research center Instituto de Telecomunicações (IT). She is pursuing a $\mathrm{PhD}$ degree in Electrical and Computer Engineering at IST. Her research interests are in the area of digital communications, with emphasis on multicarrier transmission and nonlinear distortion of digital signals.

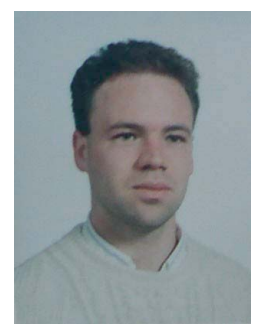

Rui Dinis (S'96 - M'00) received the $\mathrm{PhD}$ degree from Instituto Superior Técnico (IST), Technical University of Lisbon, Portugal, in 2001. From 2001 to 2008 he was a Professor at IST. Since 2008 he is teaching at FCT-UNL (Faculdade de Ciências e Tecnologia da Universidade Nova de Lisboa). He was a researcher at CAPS/IST (Centro de Análise e Processamento de Sinais) from 1992 to 2005; from 2006 to 2008 he was a researcher at ISR/IST (Instituto de Sistemas e Robótica) and in 2009 he joined the research center IT (Instituto de Telecomunicações). He has been involved in several research projects in the broadband wireless communications area. His main research interests include modulation, equalization and channel coding. 